\title{
ESTUDO ANATÔMICO DA VEIA RENAL ESQUERDA DE CADÁVERES HUMANOS BRASILEIROS
}

\author{
ANATOMICAL STUDY OF THE LEFT RENAL VEIN OF HUMAN BRAZILIANS CADAVERS
}

Pedro Duques ${ }^{1}$, Juliana R. Rodrigues ${ }^{2}$, Francisco B. da Silva Neto², Elry M. V. S. Neto² \& Elivaldo S. de Tolêdo ${ }^{3}$

\begin{abstract}
${ }^{1}$ Ex-Bolsista do Programa Institucional de Bolsas de Iniciação Científica (PIBIC). Conselho Nacional de Pesquisa (cNPq). ${ }^{2}$ Monitor. ${ }^{3}$ Docente de Anatomia. Departamento de Morfologia. Universidade Federal da Paraíba.

Correspondência: Juliana Ribeiro Rodrigues. Rua Geraldo Costa, 295/101, Manaira. 58038-130 - João Pessoa - PB.

Telefone: (83) 247-4042; (83) 9332-5521. e-mail: July_rr@medcenter.com ou heri@openline.com.br
\end{abstract}

Duques P; Rodrigues JR; Silva Neto FB; Neto EMVS \& Tolêdo ES. Estudo anatômico da veia renal esquerda de cadáveres humanos brasileiros. Medicina, Ribeirão Preto, 35: 184-191, abr./jun. 2002.

RESUMO: O estudo das variações anatômicas da veia renal esquerda (VRE) é matéria de importância na realização das anastomoses para o tratamento cirúrgico da hipertensão portal, estudos radiológicos e para as nefrectomias de doadores de transplante renal. Trata-se de um estudo observacional transversal e retrospectivo, cujo objetivo foi identificar e avaliar as variações anatômicas da VRE em cadáveres. Um total de 34 cadáveres foi analisado (24 do sexo masculino, 10 do sexo feminino) sob os seguintes aspectos: número de tributárias renais, número de veias renais acessórias, posição em relação à aorta abdominal (Ao) e à artéria renal esquerda (ARE) e comprimento da VRE. A VRE era única em 91,1\% $(n=31)$ dos casos e dupla em $8,9 \%(n=3)$. Observou-se um caso de VRE circum-aórtica. Dos 34 casos estudados, 55,8\% $(n=19)$ apresentavam duas tributárias, que se uniam para formar a VRE principal, $14,7 \%(n=5)$ apresentavam três tributárias e, em $29,4 \%(n=10)$, elas eram ausentes ( quando a VRE originase diretamente do rim, sem que fossem visualizadas, no hilo, tributárias renais, que contribuíssem para a sua formação). A veia renoazigolombar esteve presente em $26,4 \%, n=9$ (23,5\%: única; 2,9\%: dupla). A veia testicular / ovárica era única em $85,2 \%(n=29)$ e dupla em $8,8 \%(n=3)$. Em dois casos, não pôde ser estudada. Quanto à posição em relação à Ao e à ARE, a VRE encontrava-se predominantemente na topografia anterior, com variações percentuais de $44,5 \%$ a $96,5 \%$, dependendo se a ARE era única, dupla ou tripla. O comprimento médio foi de $5,4 \mathrm{~cm}$, variando de $2,2 \mathrm{~cm}$ a $8,0 \mathrm{~cm}$. Conclui-se, no presente estudo, que as tributárias renais foram mais freqüentes em número de duas; a veia renoazigolombar esteve presente em freqüência inferior à da literatura; a veia testicular / ovárica foi mais freqüente na forma única; a posição mais freqüente em relação à Ao e à ARE foi a anterior e o comprimento médio da VRE concordou com apenas duas das publicações onde tal parâmetro foi avaliado.

UNITERMOS: Veias Renais. Anatomia Regional. Rim.

\section{INTRODUÇÃO}

Segundo a clássica descrição anatômica, as veias renais são vasos que nascem no nível do hilo renal, originados pela anastomose de cinco a seis veias, seguem uma direção transversa, drenando o sangue dos rins para a veia cava inferior $(\mathrm{VCI})^{1}$. A veia renal esquerda (VRE) é mais longa que a veia renal direita, tem trajeto ventral à aorta abdominal, passando inferiormente à origem da artéria mesentérica superior. Recebe freqüentemente as seguintes tributárias: a veia supra-renal esquerda, a veia frênica inferior esquerda, a veia testicular / ovárica e, em alguns casos, a veia renoazigolombar esquerda. Apresenta desembocadura no nível da VCI, em situação discretamente mais alta que a veia renal direita 
O estudo da veia renal esquerda e suas variações encontram importância tanto quando da realização das anastomoses cirúrgicas, esplenorrenais, para o tratamento cirúrgico da hipertensão portal estudos radiológicos, como, também, nas nefrectomias de doadores para o transplante renal. Por apresentar um trajeto mais longo do rim à VCI, a VRE adquire maior importância que a veia renal direita, sendo preferida a sua utilização neste último caso. Exemplificando, de um total de 342 nefrectomias para transplante renal, realizadas em dois serviços especializados, 311 foram realizadas do lado esquerdo e apenas 31 do lado direito ${ }^{4}$.

O objetivo do presente trabalho foi identificar as variações anatômicas da VRE em cadáveres prédissecados do Setor de Anatomia Humana do Departamento de Morfologia da Universidade Federal da Paraíba (Campus 1).

\section{MATERIAL E MÉTODOS}

A amostra consistiu de 34 cadáveres mestiços, provenientes do Setor de Anatomia Humana do Departamento de Morfologia da Universidade Federal da Paraíba (Campus 1). Tratava-se de cadáveres formolizados e com cavidades abdominais pré-dissecadas, sendo $24(70,5 \%)$ do sexo masculino e $10(29,5 \%)$ do sexo feminino. Nos cadáveres, cujas estruturas anatômicas do pedículo renal não estavam bem visualiza- das, foi realizada dissecação. A veia renal esquerda foi estudada quanto aos seguintes aspectos: 1- número de tributárias provenientes do rim, que se uniam para formar a veia renal; 2- número de tributárias provenientes de outras localizações (veia supra-renal esquerda, veia frênica inferior esquerda, veia testicular/ ovárica esquerda e veia renoázigolombar); 3- número de veias renais (única ou acessórias, veias que, além da veia renal esquerda principal, partem do rim, seguindo diretamente para a veia cava inferior) e 4- posição de VRE em relação à aorta abdominal, bem como sua posição em relação à artéria renal esquerda. Foi realizada a mensuração de seu comprimento desde sua origem, no hilo renal, até a sua desembocadura na veia cava inferior, com o auxílio de uma régua milimetrada. Os dados obtidos foram tabulados para análise estatística descritiva.

\section{RESULTADOS}

Quanto ao número de veias renais, em 91,1\% $(\mathrm{n}=31)$ dos casos a VRE era única e em $8,9 \%(\mathrm{n}=3)$, a VRE era dupla. Dos 31 casos onde o vaso era único, observou-se um caso de VRE circum-aórtica, composta por um único tronco que emerge do rim e, antes de se anastomosar com a VCI, divide-se em duas veias com uma cruzando anteriormente a aorta e outra passando posteriormente (Figura 1). A veia renal dupla (acessória) pode ser observada em $8,9 \%(\mathrm{n}=3)$ casos.

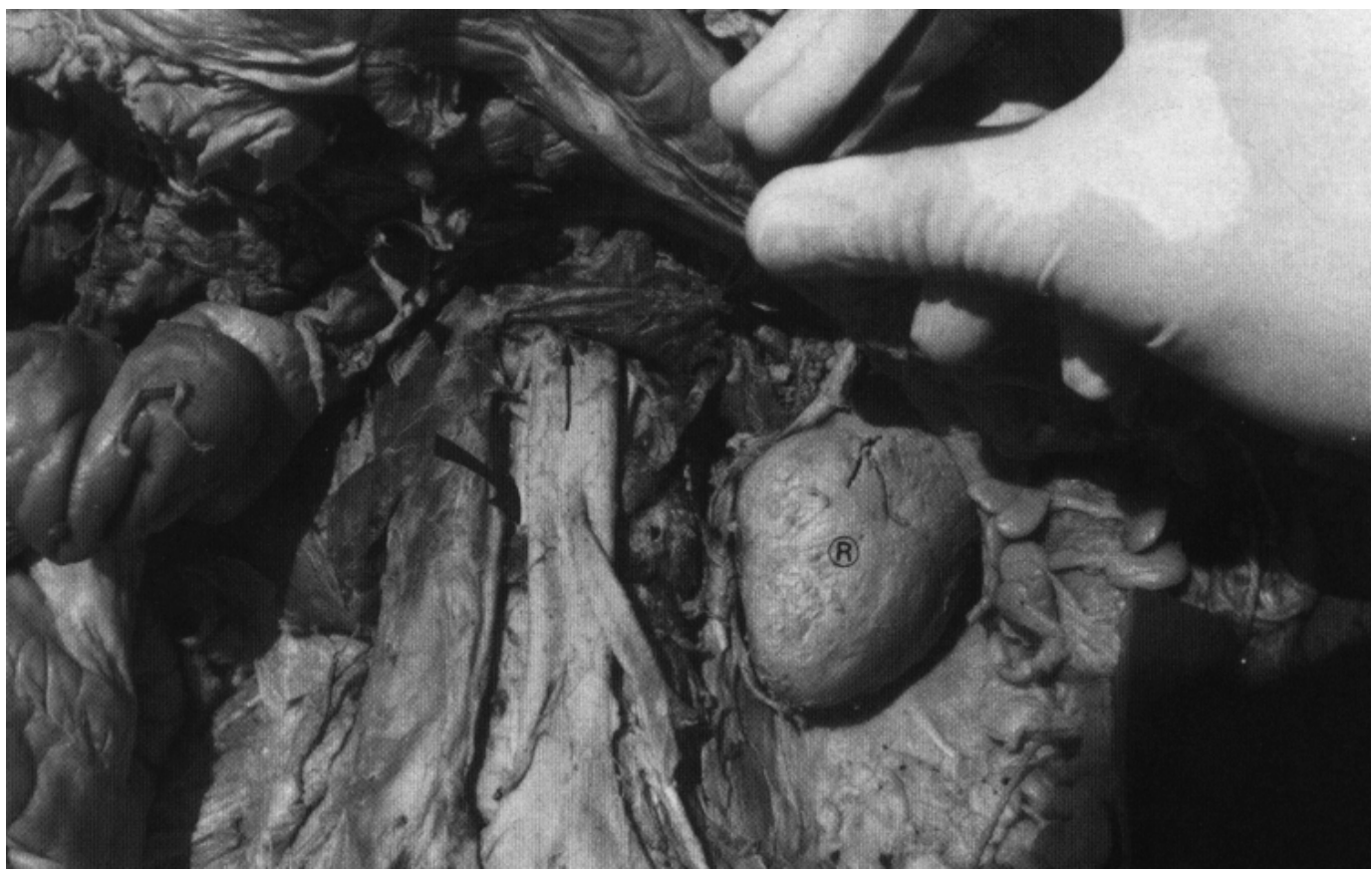

Figura 1: Peça anatômica, ilustrando veia renal esquerda, circum-aórtica, com porção dorsal (seta curva), desembocando na VCl, no nível da origem da artéria mesentérica inferior. Porção ventral (seta reta). Rim esquerdo ${ }^{\circledR}$. 
Quanto ao número de tributárias renais, que se unem para formar a VRE principal, dos 34 casos estudados, $19(55,8 \%)$ eram representados por indivíduos com duas tributárias e $5(14,7 \%)$ por indivíduos com três tributárias. Em 10 casos $(29,4 \%)$, essas tributárias renais estavam ausentes. Quanto ao número de tributárias renais, provenientes de outras localizações que não o rim esquerdo, observou-se a presença da veia renoazigolombar em 26,4\% ( $\mathrm{n}=09$ ) dos casos, sendo que, em $23,5 \%(n=08)$ das vezes, ela era única e, em apenas um caso $\left(2,9^{\circ} / \mathrm{a}\right)$ a referida veia era dupla. As veias testiculares / ováricas foram observadas em 32 dos 34 cadáveres analisados. Em 85,2\% $(\mathrm{n}=29)$ dos achados, tais veias eram únicas e, em $8,8 \%(n=3)$, eram duplas. Em dois casos, tais vasos não foram observados devido ao estado de conservação dos cadáveres. Entretanto, incluíram-se em nosso estudo por permitirem a análise de outras veias contabilizadas na pesquisa. A veia supra-renal esquerda foi apenas em número de uma e foi observada em $100 \%(n=34)$ dos casos. (Tabela I).

Quanto à posição da veia renal esquerda em relação à aorta abdominal, observou-se que, nos casos em que a veia era única $(91,1 \%, \mathrm{n}=31), 100 \%$ cruzavam a aorta por sua face anterior (um dos casos compunha a VRE circum-aórtica em que uma das porções do colar cruzava dorsalmente a aorta para desembocar na VCI). Quando a veia era dupla $(8,9 \%$, $\mathrm{n}=3$ ), $100 \%$ das vezes, elas cruzavam anteriormente a aorta. Em relação à artéria renal esquerda, que se apresentou única em 29 dos 34 cadáveres estudados, dupla em 4 e tripla em 9, obtiveram-se as seguintes frequiências em relação às posições: 1- artéria renal esquerda única: $96,5 \%(n=28)$ das VRE situavam-se anteriormente e $3,5 \%(n=1)$ situavam-se inferiormente; 2- artéria renal esquerda dupla: $50(\mathrm{n}=2)$ dos casos situavam-se anteriormente e $50 \%(\mathrm{n}=2)$ situavam-se inferiormente a ambas as ARE; 3- artéria renal esquerda tripla: $44,5 \%(\mathrm{n}=4)$ dos casos situavam-se anteriormente, 33,3\% $(\mathrm{n}=3)$ situavam-se superiormente às três ARE e 22,2\% $(n=2)$ situavam-se inferiormente. (Tabela II).

O comprimento da VRE foi aferido em todos os casos. Para os indivíduos do sexo masculino, obteve-se um comprimento médio de $6,3 \mathrm{~cm}(2,4-8,0 \mathrm{~cm})$ e para o sexo feminino de $4,6 \mathrm{~cm}(2,2-6,8 \mathrm{~cm})$. Considerando-se todos os casos em estudo, foi encontrada uma média global de $5,4 \mathrm{~cm}$, com uma variação de $2,2 \mathrm{~cm}$ a $8,0 \mathrm{~cm}$. (Tabela III).
Tabela I: D istrib uição percentual dos achados re hcionados à ve ia renale squerda e suas tỏ utárias, em 34 cadáveres do Se tor de Anatomia Humana da U riversidade Federal da Paraiba.

\begin{tabular}{|c|c|c|}
\hline Variáveis & $\mathrm{N}^{\circ}$ total & $\%$ \\
\hline Masculino & $24 / 34$ & 70,5 \\
\hline F eminino & $10 / 34$ & 29,5 \\
\hline $\begin{array}{l}\text { Veia renal esquerda } \\
\text { única* } \\
\text { dupla (acessória) }\end{array}$ & $\begin{array}{l}31 / 34 \\
3 / 34\end{array}$ & $\begin{array}{l}91,1 \\
8,9\end{array}$ \\
\hline $\begin{array}{l}\text { Tributárias renais } \\
\text { ausente } \\
\text { dupla } \\
\text { tripla }\end{array}$ & $\begin{array}{l}10 / 34 \\
1934 \\
5 / 34\end{array}$ & $\begin{array}{l}29,4 \\
55,8 \\
14,7\end{array}$ \\
\hline $\begin{array}{l}\text { Veia renoazigolombar } \\
\text { Ausente** } \\
\text { Ú rica } \\
\text { Dupla }\end{array}$ & $\begin{array}{l}25 / 34 \\
8 / 34 \\
1 / 34\end{array}$ & $\begin{array}{l}73,5 \\
23,5 \\
2,9\end{array}$ \\
\hline $\begin{array}{l}\text { Veias te sticulares ováricas } \\
\text { Úrica } \\
\text { Dupla } \\
\text { não observada }\end{array}$ & $\begin{array}{l}29 / 34 \\
3 / 34 \\
2 / 34\end{array}$ & $\begin{array}{l}85,2 \\
8,8 \\
5,8\end{array}$ \\
\hline $\begin{array}{l}\text { Veia supra-renal esquer da } \\
\text { Úrica }\end{array}$ & $34 / 34$ & 100,0 \\
\hline \multicolumn{3}{|c|}{ 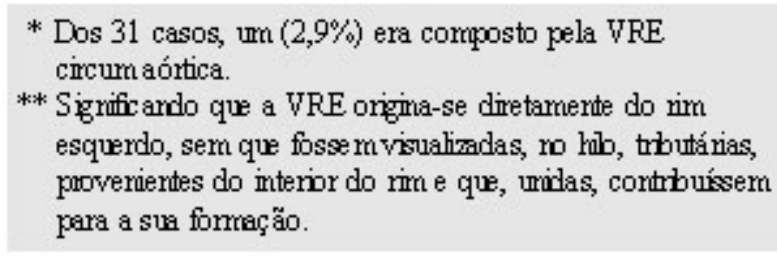 } \\
\hline
\end{tabular}

\section{DISCUSSÃO}

O reconhecimento de variações anatômicas é de importância crucial, nos procedimentos cirúrgicos que envolvem a veia renal esquerda, para prevenir lesões inadvertidas durante sua manipulação. Contribui, principalmente nas intervenções cirúrgicas, como a anastomose esplenorrenal, nas nefrectomias e em estudos radiológicos. Durante as nefrectomias esquerdas, realizadas com o objetivo de se proceder a um transplante renal, faz-se necessária a mobilização da VRE para que haja exposição das artérias renais e, 
Tahe h II. Distrb̧ uição percentual da posição da veia re tal esque rda em relação à aorta abdo mínal e à artéria re mal esquerda, em 34 cadáveres do Se tor de Anatomia Humana da U niversidade Federal da Paraiba.

\begin{tabular}{|c|c|c|c|c|c|c|c|c|}
\hline \multirow{2}{*}{ Estrutura relacionada } & \multicolumn{2}{|c|}{ Anterior } & \multicolumn{2}{|c|}{ Pasterior } & \multicolumn{2}{|c|}{ Superior } & \multicolumn{2}{|c|}{ Inferior } \\
\hline & $\mathrm{N}$ & $\%$ & $\mathrm{~N}$ & $\%$ & $\mathrm{~N}$ & $\%$ & $\mathrm{~N}$ & $\%$ \\
\hline $\begin{array}{l}\text { Aorta } \\
\text { veia úrica } \\
\text { veia duqula* }\end{array}$ & $\begin{array}{c}31 \\
3\end{array}$ & $\begin{array}{l}100 \\
100\end{array}$ & & & & & & \\
\hline $\begin{array}{c}\text { ARE** } \\
\text { Úrica } \\
\text { Dupla } \\
\text { Tripla }\end{array}$ & $\begin{array}{c}28 \\
2 \\
4\end{array}$ & $\begin{array}{c}96,5 \\
50 \\
44,5\end{array}$ & & & 3 & 33,3 & $\begin{array}{l}1 \\
2 \\
2\end{array}$ & $\begin{array}{c}3,5 \\
50 \\
22,2\end{array}$ \\
\hline
\end{tabular}

Tabeh III: Comprimento (cm)da veia renal esquerda, por sexo, em 34 cadáveres do Setor de Anatomia Humara da U riversidade Federal da Paraiba

\begin{tabular}{|l|c|c|}
\hline Sexo & Média $(\mathbf{c m})$ & Variação $(\mathbf{c m})$ \\
\hline Masculino & 6,3 & $2,4-8,0$ \\
\hline Femirinino & 4,6 & $2,2-6,8$ \\
\hline Total & $\mathbf{5 , 4}$ & $\mathbf{2 , 2 - 8 , \theta}$ \\
\hline
\end{tabular}

em seguida, realize-se a ligadura e secção da artéria. O procedimento de mobilização inicia-se pela ligação e secção de tributárias como as veias testicular/ ovárica, supra-renal e renozigolombar ${ }^{4,5}$. Nas mais diversas situações, as tributárias podem variar quanto ao padrão de drenagem, exigindo maior habilidade técnica, o que envolve seu reconhecimento e estudo.

Segundo Baptista-Silva et al, $1997^{4} \mathrm{em}$ análise de 311 nefrectomias esquerdas para transplante renal, $65,27 \%$ das VRE apresentavam três tributárias (veia supra-renal, veia testicular/ovárica e veia renoazigolombar) ao passo que $34,72 \%$ das VRE apresentavam duas tributárias (veias supra-renal e testicular/ ovárica). Neste estudo, as tributárias que sempre drenavam o sangue para a VRE eram a veia suprarenal e a veia testicular/ ovárica. Na presente observação, a veia testicular/ ovárica era única em $85,2 \%$ $(\mathrm{n}=29)$ das vezes, comparando-se com $8,8 \%(\mathrm{n}=3)$, em que se apresentavam na forma dupla, isto é, quando duas tributárias desembocavam diretamente na VRE. Baptista-Silva et al., $1997^{4}$ observaram a VRE recebendo a veia testicuiar/ ovárica em $100 \%$ das vezes, enquanto que, no presente estudo, observou-se que a mesma recebia a veia testicular/ovárica em $94 \%$ das vezes. No restante dos casos $(5,8 \%)$, não foi possível observá-la devido ao estado de conservação dos cadáveres.

A veia supra-renal esquerda recolhe o sangue da glândula supra-renal, descrevendo trajeto descendente, até penetrar na VRE Como as demais tributárias da VRE, tal estrutura também pode ser alvo de variações. Mas et al., $1987^{5}$ identificaram 3 casos $(2,7 \%)$ de veia supra-renal dupla, de 110 nefrectomias para transplante, em que 78 foram realizadas à esquerda. Dos 34 cadáveres analisados nesta pesquisa, $100 \%$ das veias supra-renais esquerdas eram únicas e desembocavam na VRE, portanto, não apresentavam variações anatômicas.

O sistema de veias tributárias, do qual a veia renoazigolombar participa, dificulta a dissecação e as manobras de mobilização da veia renal durante as nefrectomias para transplante. Pelo fato de a veia renoazigolombar ser muito curta, mantém fixa a VRE, dificultando sua dissecação. Ainda assim, é um vaso extremamente frágil, podendo provocar sangramento profuso de difícil localização. A veia renoazigolombar esteve presente em número de uma ou duas veias. $\mathrm{Na}$ literatura, ela apresenta freqüência variável de aparecimento Lejars, $1888^{7}$ identificou tal vaso em 88\% dos casos, Cooley \& Wukasch, $1978 \mathrm{~cm} \mathrm{95 \%}$ dos casos e, em percentagens menores, Yang et al., $1992^{9}$ e Pinto, $1996^{10}$, a identificaram em $60,8 \%$ e $3,36 \%$, respectivamente. No atual estudo, a veia reiioazigolombar esteve presente em $26.4 \%$ ( $n=9$ ) dos 
cadáveres estudados. Deste percentual, em 23,5\% $(\mathrm{n}=8)$ a veia era única e, em apenas um caso $(2,9 \%)$, a veia era dupla, dados que demonstram a baixa freqüência de aparecimento dessa estrutura, se comparada com a literatura.

Segundo Hollinshead \& Rosse $1991^{11}$ o aparecimento de veias renais múltiplas é quase tão freqüente como as artérias renais múltiplas do lado direito. Em contrapartida, são raras as do lado esquerdo. Na presente observação, 91,1\% ( $\mathrm{n}=31)$ das VRE estudadas eram únicas, isto é, apenas um vaso fazia o percurso do hilo renal até a VCI. Observaram-se 3 casos $(8,9 \%)$ de VRE dupla (acessória), ou seja, quando duas veias partiam de locais distintos do hilo renal e desembocavam na VCI, também em pontos diferentes. Bertoliazo et al., $1981^{12}$ relataram um caso de veia renal dupla, sendo uma superior e outra inferior, dentre os 202 cadáveres dissecados. Mas et al $1987^{5}$ identificaram $11.8 \%(11=13)$ de VRE dupla e $(n=6)$ de VRE tripla.

As principais descrições de importância, na literatura, quanto às variações anatômicas da VRE relacionam-se à posição desse vaso em relação à aorta abdominal, originando a VRE retroaórtica e circumaórtica. Durante a oitava semana de vida fetal, as veias cardinais posteriores se obliteram à direta e à esquerda e, a VCI se origina a partir da anastomose subsupracardinal direita. A veia renal ventral se forma a partir da persistência da anastomose subcardinal ou intersubcardinal e irá constituir a VRE ${ }^{13}$. Quando ocorre a persistência isolada da anastomose subsupracardinal e obliteração da anastomose subcardinal anterior, o resultado será a formação de uma VRE retroaórtica única ${ }^{14}$.

Na embriogênese da VCI, as veias supracardinais se comunicam livremente umas com as outras e com as veias subcardinais, na proximidade da anastomose intersubcardinal. A mais cranial das conexões entre as supracardinais, juntamente com as anastomoses supracardinal - subcardinal e intersubcardinal completam um anel venoso em torno da aorta, situado abaixo da origem da artéria mesentérica superior, denominado colar renal ${ }^{15}$. A VRE periaórtica ou circumaórtica é, portanto, o resultado da obliteração da veia renal dorsal esquerda e da anastomose intersupracardinal, associada à persistência da anastomose subsupracardinal esquerda ${ }^{13}$.

Dos 311 rins esquerdos, estudados por Baptista Silva et al., $1997^{4}$ observou-se a presença de VRE retroaórtica em 1,28\% ( $n=4)$ dos casos. Lattore, $1993^{16}$ e Pinto, $1996{ }^{10}$ observaram a ocorrência deste vaso $\mathrm{cm} 3,4 \%$ e $1,98 \%$, respectivamente. Bertollazzo et al. $1981^{12}$ observaram tal vaso em apenas um caso $(0,44 \%)$ dos 225 cadáveres analisados. Silva et al., $1992^{3}$ analisando 55 cadáveres, encontraram a VRE retroaórtica em 7,2\% $(\mathrm{n}=4)$ dos casos. Thomas, $1970^{17}$ Hoeltl et al.,1990 ${ }^{18}$ observaram esse vaso em $3 \%$ a $10 \%$. Le Bouëdec et al., 2000 ${ }^{14}$, analisando 103 exames abdôminopélvicos por ressonância nuclear magnética, encontraram a VRE retroaórtica em 5,8\% $(\mathrm{n}=6)$ dos casos. Pick \& Anson , $1940^{19}$ observaram uma freqüência de 3,46\% (7/202), Reis \& Esenther, $1959^{21}$ em 1,2\% (6/500) e Davis \& Lundberg, $1968^{21}$ em 3,3\% dos casos. Royster et al., $1974^{22}$, estudando 228 pacientes operados de aneurisma da aorta abdominal e 159 cadáveres, encontraram 5 casos $(1,2 \%)$ de VRE retroaórtica, um caso $(0,2 \%)$ de VRE dupla e retroaórtica e um caso $(0,2 \%)$ de VRE dupla, ambas as veias anteriores à aorta. Por fim, dos 38 cadáveres estudados por Gotta et al., 1999 observaram-se 3 casos dessa variação anatômica. No presente estudo, não observamos a VRE única ou dupla retroaórtica.

A veia renal circum-aórtica, anel periaórtico ou colar renal também é uma variante anatômica de achado pouco freqüente. No estudo realizado por BaptistaSilva et al., $1997^{4}$ dos 311 rins esquerdos, dissecados para transplante renal, observaram-se apenas 3 casos de VRE circum-aórtica $(0,96 \%)$. Ressalte-se que, nesse estudo, a duplicação de VCI esteve presente em dois casos $(0,64 \%)$. Pinto, 1996 observou a sua presença em 1,98\% dos casos. Dos 55 cadáveres analisados por Silva et al., observou-se a VRE circumaórtica em 1 caso $(1,8 \%)$. Já com casuística maior, 500 casos, Reis \& Esenther, $195^{920}$ observaram seis casos $(1,2 \%)$ de VRE circum-aórtica. Davis \& Lundberg, $1968^{21}$ observaram um percentual semelhante para tal variação anatômica (1,5\%). Mas et al. $1987^{5}$ identificaram dois casos $(1,1 \%)$. Na casuística de 387 pacientes estudados por Royster et al., 197422, observou-se um caso $(0,2 \%)$ de colar renal, indicando um dos mais baixos percentuais de ocorrências dessa variação anatômica já descritos na literatura. No presente estudo, dos 34 cadáveres analisados, a VRE circum-aórtica foi observada em um caso (2,9\%). Identificou-se que a porção dorsal à aorta penetrava na VCI, no nível da origem da artéria mesentérica inferior, como descrito previamente para um caso de VRE retroaórtica ${ }^{22}$.

A Tabela IV indica as frequiências de ocorrência da VRE circum-aórtica em sete estudos descritos anteriormente neste trabalho com o acréscimo dos 
nossos resultados. Pode-se observar que a freqüência de $2,9 \%$, notificada na presente observação, equipara-se aos resultados de quatro estudos na literatu$\mathrm{ra}^{3,5,10,20,21}$. Em comparação aos estudos de Royster et al.,197422 Baptista-Silva et al., 19974, observou-se que a frequiência de ocorrência encontrada no presente trabalho era mais elevada. Assim, os resultados do presente trabalho encontram-se embasados nas cinco publicações anteriormente mencionadas.

\begin{tabular}{|c|c|c|}
\hline Estudos & $n$ & $\%$ \\
\hline REIS et al (1959) & 500 & 1,2 \\
\hline DAVIS et al (1968) & 207 & 1,5 \\
\hline ROYSTER et al(1974) & 387 & 0,2 \\
\hline MAS et al (1987) & 78 & 1,1 \\
\hline SILVA et al (1992) & 55 & 1,8 \\
\hline PINTO (1996) & 101 & 1,98 \\
\hline BAPTISTA-SILVA et al (1997) & 311 & 0,96 \\
\hline Presente estudo & 34 & 2,9 \\
\hline
\end{tabular}

Em relação ao número de veias tributárias renais, que se unem para formar a veia renal principal e sua posição em relação à emergência no rim, Satyapal, $1995^{23}$ propôs uma classificação de grande utilidade para os diferentes padrões de drenagem da VRE, mas, que, no presente trabalho, não foi adotada. Nesta pesquisa, foram observadas tributárias renais em número de duas em 19 dos 34 casos estudados $(55,8 \%)$ e em número de 3 em 5 dos 34 casos (14,7\%). Nos 10 casos restantes $(29,4 \%)$, as tributárias renais, que se uniriam no hilo para formar a VRE principal, estavam ausentes (Tabela I).

Os dados referentes à posição da VRE em relação à aorta abdominal e à artéria renal esquerda são apresentados na Tabela II. Em relação à aorta abdominal, observou-se que $100 \%$ dos casos tinham seu trajeto anterior à aorta, excetuando-se o achado de VRE circum-aórtica em que urna das tributárias formadoras possuía trajeto dorsal à aorta. Quanto à posição em relação à artéria renal esquerda, quando era vaso único, 96,5\% situavam-se anteriormente e 3,5\%, inferiormente. Quando a artéria era dupla, 50\% das VRE eram anteriores e 50\% eram inferiores a ambas as ARE. Quando a artéria era tripla, 44,5\% das veias eram anteriores, $33,3 \%$ eram superiores às três ARE e $22,2 \%$ estavam em posição inférior a essas mesmas artérias. Tal distribuição reflete a variabilidade no posicionamento da VRE em relação às estruturas adjacentes e, na presente pesquisa, observou-se que a VRF era predominantemente anterior à aorta e à artéria renal esquerda, mesmo quando ela possuia mais de dois ramos (artéria renal tripla).

O comprimento da VRE tem sido outro aspecto anatômico, estudado pelos especialistas no assunto. Anson \& Doseler et al., $1961^{24}$ observaram uma variação no comprimento de $6 \mathrm{~cm}$ a $11 \mathrm{~cm}$, com média de $8,4 \mathrm{~cm}$. Gotta et al., $1999^{13}$ observaram que, em média, o comprimento situava-se em torno de $5,9 \mathrm{~cm}$. Do grupo de cadáveres estudados na presente pesquisa (Tabela III), obteve-se urna média global para o comprimento de $5,4 \mathrm{~cm}$ com variação entre $2,2 \mathrm{~cm}$ e $8,0 \mathrm{~cm}$. Identificamos que a VRE em média foi $1,7 \mathrm{~cm}$ maior no sexo masculino que no feminino. Os resultados de média global do comprimento, quando comparados com os de Anson \& Doseler 1961 24 , indicam que as VRF avaliadas na presente observação foram $3,0 \mathrm{~cm}$ menores. Entretanto, encontramos concordância quando comparadas com a média global de $5,9 \mathrm{~cm}$, observada por Gotta et al $1999^{13}$.

\section{CONCLUSÕES}

Dos resultados obtidos, conclui-se que: 1 - a VRE única foi o achado mais freqüentemente observado $(91,1 \%)$ na população estudada; 2 - em relação à formação da VRE principal, para, em seguida, desembocar como vaso único na VCI, as tributárias renais estavam presentes na maior parte das ocasiões, sendo em $55,8 \%$, em número de duas; 3 - a veia renoazigolombar esteve presente em freqüência inferior $(26,4 \%)$ à relatada na literatura, sendo, na maior parte das vezes única; 4 - a veia testicular/ ovárica foi mais freqüente na forma única $(85,2 \%)$; a veia suprarenal esquerda esteve presente em número de uma tributária em 100\% dos casos; 5 - em relação à aorta abdominal e à artéria renal esquerda, a VRE esteve predominantemente em posição anterior; 6 - o comprimento médio da VRE foi de $5,4 \mathrm{~cm}$, concordando apenas com uma das duas publicações onde tal parâmetro foi avaliado. 
DUQUES P; RODRIGUES JR; SILVA NETO FB; NETO EMVS \& TOLÊDO ES. Anatomical study of the left renal vein of human brazilians cadavers. Medicina, Ribeirão Preto, 35: 184-191, apr./june 2002.

ABSTRACT: The study of the anatomical variations of the left renal vein (LRV) is an important subject to the accomplishment of the anastomosis to the surgical treatment of the portal hypertension, to the radiological studies and to the nephrectomies of the renal transplantation donors. This is a retrospective; transversal and observational study whose objective was to identify and study the anatomical variations of the LRV in cadavers. We have analyzed 34 cadavers (24 male; 10 female) under the following aspects: number of the tributaries renals, number of the accessories renals, position in relation to the abdominal aorta (Ao) and in relation to the left renal artery (LRA) and length of the LRV. The LRV was single in $91,1 \%$ of the cases and double in $8,9 \%(n=3)$. It was observed one case of circumaortic LRV. Among the 34 studied cases, $55,8 \%(n=19)$ showed two tributaries which joined to form a principal LRV, $14,7 \%(n=5)$ showed three tributaries and in $29,4 \%(n=10)$ they were absent (to mean when the LRV is originated directly from left kidney without be seen tributaries, on the hile, that contribute to its formation). The renolumbar vein was present in $26,4 \%$ (n (23,5\% single; $2,9 \%$ double.). The testicular / ovarian vein was single in $85,2 \%$ $(n=29)$ and double in $8,8 \%(n 3)$. In two cases it could not be studied. In relation to the position of the Ao and to the LRA, the LRV is basically found in the anterior topography with percent variations of $44,5 \%$ to $96,5 \%$, depending if the LRA was single, double, or triple. The average length was of $5,4 \mathrm{~cm}$ varying from $2,2 \mathrm{~cm}$ to $8,0 \mathrm{~cm}$. In this study we came to the conclusion that the renal tributaries were more frequently two; the renolumbar vein was present in a frequency inferior to the one in the literature; the testicular/ovarian was more frequent in the single form; the more frequent position in relation to the Ao and to the LRA was the anterior and average length of the LRV was in accordance only with two of the publications in which this parameter was analyzed.

UNITERMS: Renal Veins. Anatomy, Regional. Kidney.

\section{REFERÊNCIAS BIBLIOGRÁFICAS}

1 -TESTUT L \& LATARJET A. Venas aorticas. In: TESTUT L \& LATARJET A, eds. Compendio de anatomia descriptiva, Saivat Editores, Mallorca, Barcelona, p. 304-327, 1968.

2 - GRAY HFRS \& GOSS CM. As veias do abdome e pelve. In: GOSS CM. Gray anatomia, 29a. ed, Guanabara Koogan, Rio de Janeiro, p. 581-583, 1988.

3- SILVAAL; REIS TD; VARONI P; NETO JBR \& MERCHEDHT. Estudo anatômico da veia renal esquerda: sua aplicação à anastomose esplenorrenal. Folha Méd 105: 3-6, 1992.

4- BAPTISTA-SILVAJCC; VERISSIMO MJ; CASTRO MJ; CÂMARA ALG \& PESTANA JOM. Anatomical study of the renal veins observed during 342 living- donor nephrectomies. Med J 115: 1456-1459,1 997.

5- MAS AG, ARANGO O, GUZMÁN A, CORONADO J, ROSALES A; CORTADELLAS R \& BALLESTEROS JJ. Anatomia hilar venosa renal. A propósito de 110 nefrectomias para transplante. Actas Urol Esp 11: 172-175, 1987.

6 - GRAY HFRS. Esplancnologia. In: WILLIANS PL; WARWICK R; DYSON M \& BANNISTER LH. Gray anatomia, 37a・ ed, Guanabara Koogan, Rio de Janeiro, v. 2, p. 1379-1383, 1995.

7 - LEJARS E. Les voies de surete de la veine renale. Buli Soc Anat, Paris, 63: 504-511, 1888.
8 - COOLEY DA \& WUKASCH DS. Techniques in vascular sur gery. In: COOLEY DA \& WUKASCH DS, eds Techniques in vascular surgery, W. B. Saunders, Philadelphia, p. 1-282, 1978.

9- YANG SC; SUH DH KIM YS \& PARK K. Anatomical study of the left renal vem and its draining veins, as found during living donor nephrectomy. Transplant Proc 24: 1333-1334, 1992.

10- PINTO MG. Variações anatômicas venosas renocava en contradas em 101 cadáveres. Dissertação de Mestrado, Escola Paulista de Medicina da UNIFESP, São Paulo. p. 1104, 1996.

11 - HOLLINSHEAD WH \& ROSSE C. Parede abdominal posterior e órgãos associados. In. HOLLINSHEAD WH \& ROSSE C, eds. Anatomia, 4 ed, Editora Interlivros, Rio de Janeiro, p. 601-602, 1991.

12 - BERTOLAZZO W; ROMERO AM; BICA DTG; CAVALHEIRO FCR; FILHO FB; ARANHA NC \& GONÇALVES CRP. Variação anatômica da veia renal esquerda. Seu trajeto retroarterial aórtico. Rev Bras Cir 71: 301-304, 1981.

13- GOTTA E; N'GUESSAN G; GNAGNE '\{; KASSANDOU 5 \& KOKOUAA. La veine rénale gauche retro-aortique: à propos de trois cas. Morphologie 83: 47-50, 1999. 
14 - LE BOUEDEC G; BAILLY C; VINCEN C; POMEL C; BETHMONT T \& DAUPLAT J. La veine rénale gauche retro-aortique. Presse Med 29: 1034, 2000.

15- HUNTINGTON GS. The morphology of the pulmonary artery in the mammalia. Anat Rec 17: 165-202, 1920.

16- LATTORE J. Malformaciones congenitas. In: VILLALONGA JT, eds. Sector iliocava, Uriach, Barcelona, p. 70-112, 1993.

17- THOMAS TV. Surgical implications of retroaortic left renal vem. Arch Surg 100: 738-740, 1970.

18 - HOELTL W, HUBY W \& AHARINEJAD S. Renal vem anatomy and its implications for retroperitoneal surgery. J Urol 143: 1108-1114, 1990.

19- PICK JW \& ANSON BJ. The renal vascular pedicle. J Urol 44: 411-434, 1940.

20 - REIS RH \& ESENTHER G. Variations in the pattern of renal vessels and their relation to the type of posterior vena cava in man. Am J Anat 104: 295-318, 1959. Lstudo anatômico da veia rena! esquerda $\mathrm{cm}$ cadáveres humanos hrasilciros
21 - DAVIS CJ \& LUNDBERG GD. Retroaortic left renal vem: a relatively frequent anomaly. Am J Clin Pathol 50: 700-703, 1968.

22- ROYSTER TS, LACEY L \& MARKS RA. Abdominal aortic surgery and the left renal vem Am J Surg 127: 552-554, 1974.

23 - SATYAPAL KS. Classification of the drainage patterns of the renal veins. J Anat 186 (PT2): 329-333, 1995.

24-ANSON BJ \& DOSELER EH. Common variations in renal anatomy affecting blood supply. Form and topography. Surg Gynecol Obstet 112: 439-449, 1961.

Recebido para publicação em 12/04/2001

Aprovado para publicação em 25/06/2002. 